\title{
Intrathoracic malignant peripheral nerve sheath tumors: imaging features and implications for management
}

\author{
Sophia Constance Kamran ${ }^{1,2,4}$, Atul Bhanudas Shinagare ${ }^{1,2,4}$, \\ Stephanie Anne Holler Howard ${ }^{1,2,4}$, Mizuki Nishino ${ }^{1,2,4}$, Jason Laurence Hornick ${ }^{3,4}$, \\ Katherine Margaret Krajewski ${ }^{1,2,4}$, Nikhil Himmatsinh Ramaiya ${ }^{1,2,4}$

\footnotetext{
${ }^{1}$ Department of Imaging, Dana-Farber Cancer Institute, 450 Brookline Ave, Boston, MA 02115, USA

${ }^{2}$ Department of Radiology, Brigham and Women's Hospital, 75 Francis Street, Boston, MA 02115, USA

3 Department of Pathology, Brigham and Women's Hospital, 75 Francis Street, Boston, MA 02115, USA

${ }^{4}$ Harvard Medical School, Boston MA 02115, USA
}

Radiol Oncol 2013; 47(3): 230-238.

Received 11 January 2013

Accepted 5 April 2013

Correspondence to: Atul B. Shinagare, MD, Department of Imaging, Dana-Farber Cancer Institute, 450 Brookline Ave, Boston, MA 02115, USA. Phone: +1 617650 7465; Fax: + 617582 8574; E-mail: ashinagare@partners.org

Disclosure: No potential conflicts of interest were disclosed. In financial disclosures the authors stated that the investigator Mizuki Nishino $(\mathrm{MN})$ had been supported by $1 \mathrm{~K} 23 \mathrm{CA} 157631(\mathrm{NCl})$ from the National Institutes of Health.

Background. The aim of the study was to analyze the clinical and imaging characteristics of primary intrathoracic malignant peripheral nerve sheath tumors (MPNSTs).

Patients and methods. In this institutional review board (IRB)-approved retrospective study, clinical and imaging features of 15 patients (eight men; mean age 50 years [range 18-83)] with pathologically proven malignant peripheral nerve sheath tumors seen from January 1999 to December 2011 were analyzed. Imaging features (CT in 15, MRI in 5 and PET/CT in 4) of primary tumors were evaluated by three radiologists and correlated with clinical management.

Results. Of the 15 tumors, six were located in the mediastinum (two each in anterior, middle and posterior mediastinum), four in chest wall, two were paraspinal, and three in the lung. Four patients had neurofibromatosis-1 (NF1); four tumors had heterologous rhabdomyoblastic differentiation (malignant triton tumor). Masses typically were elongated along the direction of nerves, with mean size of $11 \mathrm{~cm}$. The masses were hypo- or isodense to muscles on CT, isointense on T1-weighted images, hyperintense on T2-weighted images and intensely fluorodeoxyglucose (FDG) avid (mean standardized uptake value [SUV] $]_{\max }$ of 10.5 [range 4.4-23.6]). Necrosis and calcification was seen in four tumors each. Finding of invasion of adjacent structures on imaging led to change in management in seven patients; patients with invasion received chemoradiation.

Conclusions. Intrathoracic MPNSTs appear as large elongated masses involving mediastinum, lung or chest wall. Radiological identification of invasion of adjacent structures is crucial and alters therapy, with patients with invasion receiving neoadjuvant or adjuvant chemoradiation.

Key words: malignant peripheral nerve sheath tumors; chest; neurofibromatosis; imaging; malignant triton tumor

\section{Introduction}

Malignant peripheral nerve sheath tumors (MPNSTs) are rare, accounting for $5-10 \%$ of all soft tissue sarcomas. ${ }^{1}$ MPNSTs usually demonstrate nerve sheath differentiation, and although they grossly appear similar to benign peripheral nerve sheath tumors such as schwannomas and neurofibromas, MPNSTs commonly contain hyperchromatic spindle cells with nuclear atypia, mitotic activity, and areas of tumor necrosis on histology. ${ }^{1,2}$ Rapid enlargement, pain, or associated neurological symptoms favor MPNST over a benign nerve sheath tumor. ${ }^{1}$ MPNSTs are most often sporadic, 

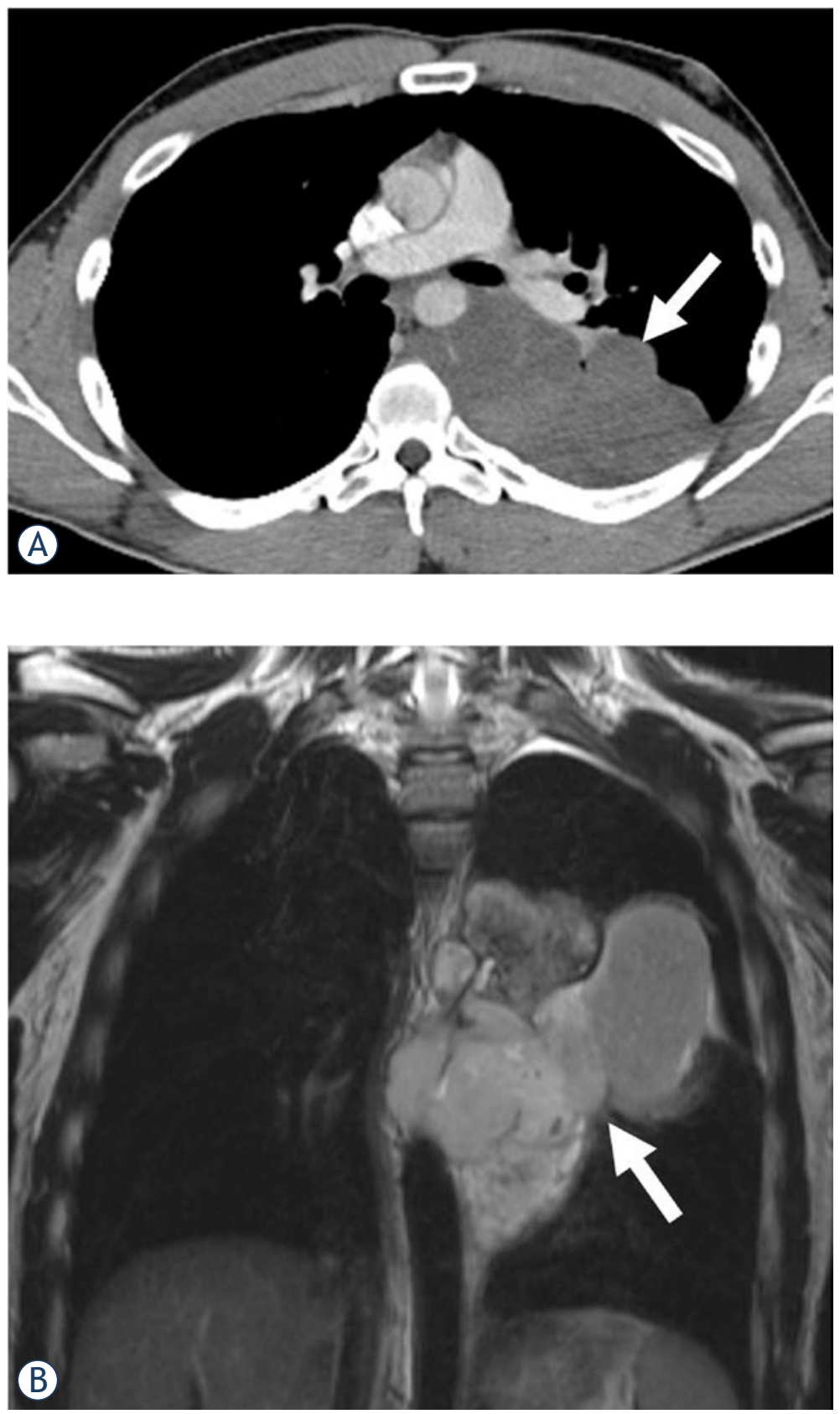

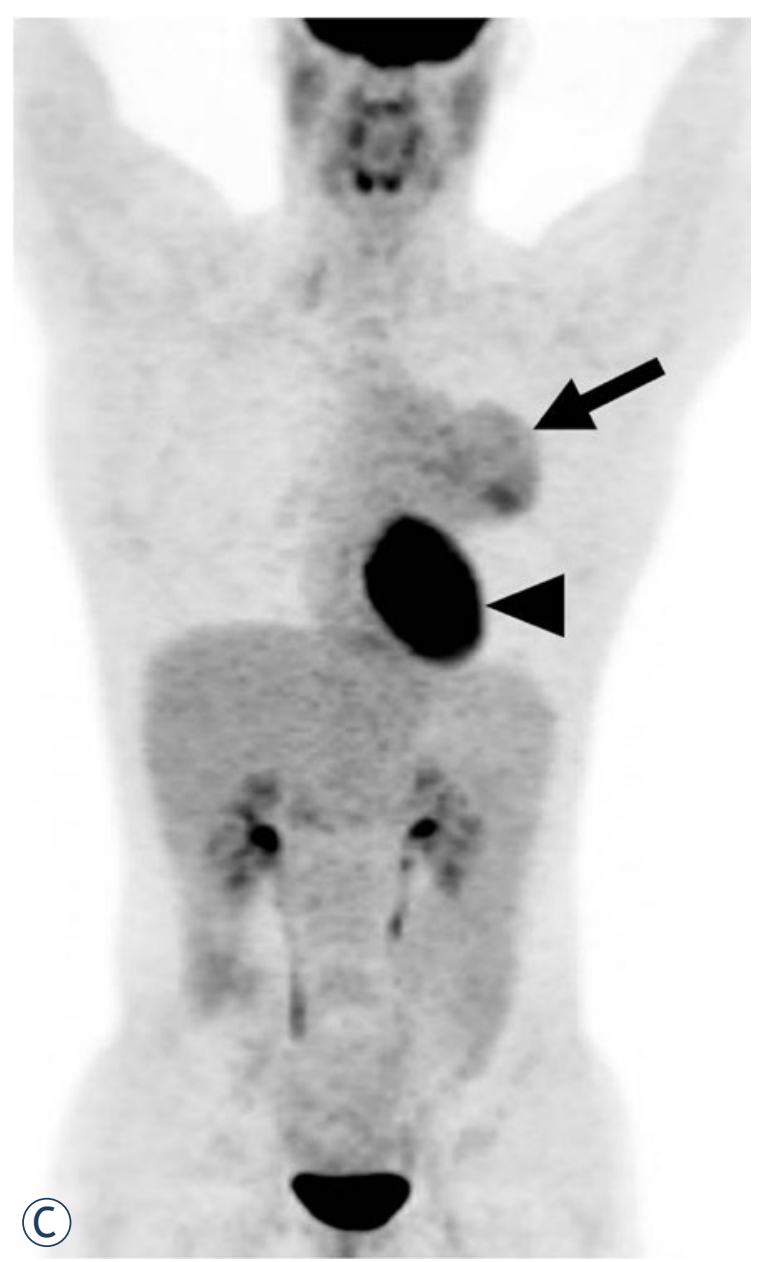

FIGURE 1. A 31-year-old man with mediastinal malignant peripheral nerve sheath tumor. A. Axial contrast-enhanced CT (CECT) shows a low attenuating mildly enhancing posterior mediastinal mass (arrow) displacing the aorta, azygous vein and esophagus. The mass encompasses more than $50 \%$ circumference of the descending thoracic aorta. B. Coronal T2-weighted MR image shows a heterogeneous lobulated hyperintense mass (arrow). C. Coronal maximum intensity projection (MIP) PET image shows a moderately fluorodeoxyglucose (FDG) avid mediastinal mass (arrow, standardized uptake value $[S U V]_{\max } 5.2$ ). Physiologic activity is noted in the heart (arrowhead). although $20-30 \%$ occur in association with neurofibromatosis-1 (NF1). ${ }^{1}$ Patients typically present around age $40-50^{1}$, although patients with NF1 often present earlier (mean age 30 years). ${ }^{3}$ MPNSTs most often occur in the extremities ${ }^{1}$, followed by trunk and head and neck, however MPNSTs can arise anywhere in the body. Intrathoracic MPNST are particularly rare. ${ }^{4}$ Pathologically, MPNSTs typically have a fascicular growth pattern and are composed of spindle cells with variable nuclear atypia and mitotic activity. MPNSTs with heterologous rhabdomyoblastic (i.e. skeletal mus- cle) differentiation are called malignant triton tumors (MTTs). MPNSTs have an aggressive clinical course with 5- and 10-year survival rates of 34-60\% and $22-45 \%$ respectively. ${ }^{1,5}$ MTT is associated with an even worse clinical outcome, with a 5-year survival rate of $11-15 \% .{ }^{6}$ Location also plays a role in outcome, with tumors in the trunk associated with poorer prognosis, likely attributable to difficulty in achieving negative surgical margins and consequent local or regional recurrence. ${ }^{1,5}$ While studying the impact of rhabdomyoblastic differentiation (MTT), presence of NF1 and location on the out- 


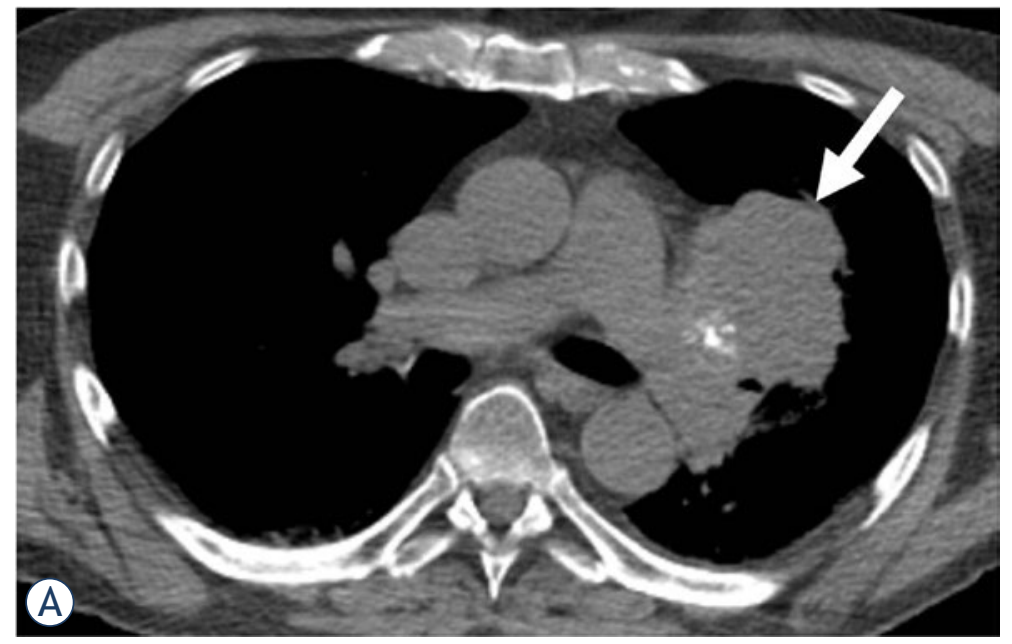

FIGURE 2. A 67-year-old man with MPNST involving the left lung. A. Unenhanced $\mathrm{CT}$ image of the chest at the level of main pulmonary arteries shows a lobulated mass abutting the left hilum with peripheral foci of calcification (arrow). B. Coronal maximum intensity projection (MIP) image from PET/CT shows an intensely fluorodeoxyglucose (FDG) avid mass (arrow, standardized uptake value $[S U V]_{\max }$ 23.6). Patient underwent left pneumonectomy on which intrapulmonary location of the mass was confirmed. Rhabdomyoblastic differentiation (malignant triton tumor) was noted on pathology.

come of 84 consecutive patients with $\mathrm{MPNST}^{7}$, we noticed that the imaging literature on intrathoracic MPNST is extremely limited, consisting mostly of case reports with little information on imaging findings. ${ }^{4,8-14}$ Therefore, the purpose of this study was to assess the clinical and imaging characteristics of primary intrathoracic MPNST.

\section{Patients and methods}

\section{Patients}

In this institutional review board-approved study, the electronic pathology patient database was reviewed to identify patients with pathologically proven primary intrathoracic MPNSTs (mediastinum, lung, and chest wall tumors) evaluated at our institution from January 1999 to December 2011. First, we identified a total of 110 patients with pathologically proven MPNSTs. Among them, 26 patients were excluded because they were referred from outside institution and the pathology of the primary tumor was not confirmed by review at our institution. Among the remaining 84 patients, 21 patients had intrathoracic MPNSTs. Of these 21 patients, 6 were excluded because imaging of the primary tumor was not available. Therefore, the final study population consisted of 15 patients with pathologically proven intrathoracic MPNST

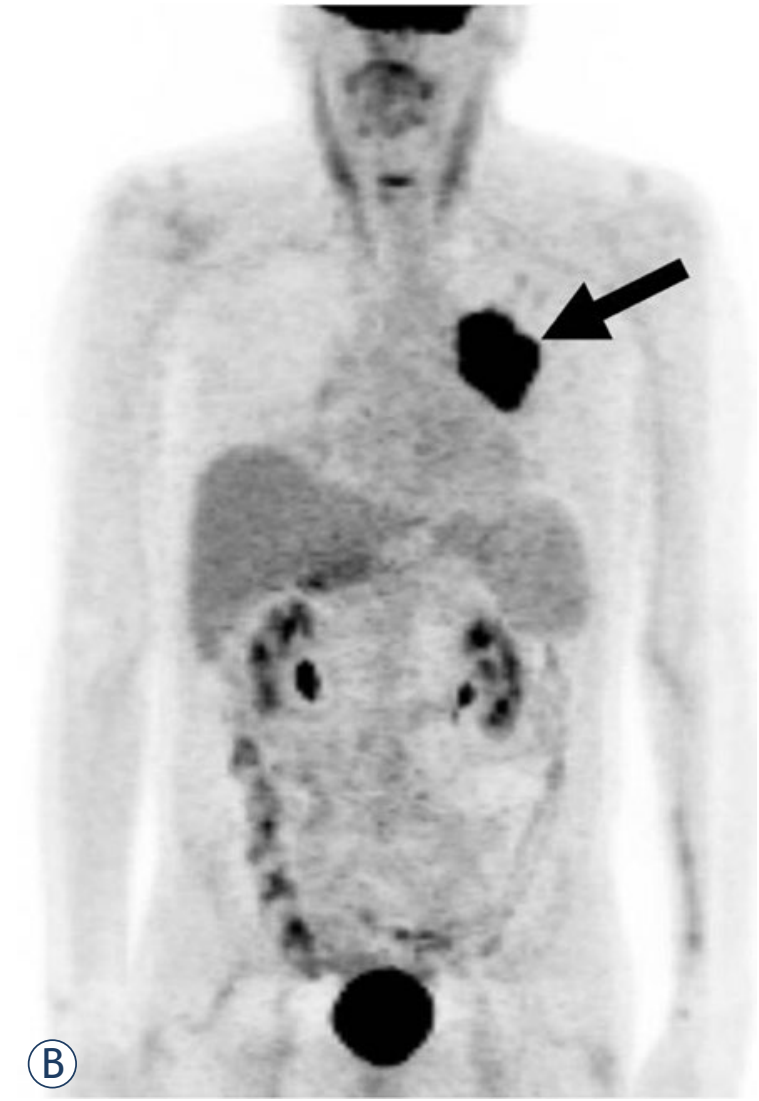

who had imaging of primary tumors available for review. Mediastinal tumor localization was determined by an adaptation of the Felson methodology. For large tumors, we used the epicenter of the tumor to define its location. Clinical features including clinical presentation and treatment were correlated with imaging findings.

\section{Image Analysis}

All imaging analysis was performed, in consensus, by three radiologists (N.R., S.H. and A.S.) with 13, 10 and 7 years of experience. Images were reviewed on the picture archiving and communication system (PACS) using commercially available workstations (Centricity, General Electric, Barrington, IL).

A systematic review of available imaging studies was performed and location, size (single longest dimension was selected by manually placing caliper on the axial or coronal images), and imaging features (CT in 15, MRI in 5 and PET/CT in 4) of the primary tumors were recorded. CT attenuation of the tumor was compared to muscles by placing a circular region of interest (ROI) in the most representative portion of the tumor. Image inten- 
sity on $\mathrm{T} 1$ and $\mathrm{T} 2$-weighted images was visually compared to muscles. Degree of enhancement was evaluated in comparison with skeletal muscle and graded as mild if less enhancement than skeletal muscle, moderate if similar degree of enhancement as muscle (considered similar for the purpose of this study if the ROI values were within $10 \%$ the muscle), and intense if more enhancement than muscle using ROI measurements, similar to previous reports. ${ }^{15}$ Homogeneous or heterogeneous appearance of the tumors on CT and MRI, presence of hemorrhage (defined as areas that are hyperdense on unenhanced CT images or T1-hyperintense areas on T1-weighted MR images), necrosis (defined as hypodense and/or T2-hyperintense, non-enhancing area within the tumor), and calcifications were also recorded. Degree of fluorodeoxyglucose (FDG) uptake was measured on PET/CT images by manually placing a circular ROI over the most FDG avid portion of the lesion on a commercially available workstation (Hermes, Hermes Medical Solutions Inc, Greenville, NC, USA). Standardized uptake value $(\mathrm{SUV})_{\max }$ was used to represent the FDG uptake of the tumor. Invasion of adjacent structures, seen as extension of tumor invading and distorting the adjacent tissues, was noted and its impact on management was studied.

Differentiation between malignant and benign nerve sheath tumors is challenging, especially in NF1 patients. Signs of malignancy in this context on imaging include high attenuation and necrosis/ hemorrhage on $\mathrm{CT}$, heterogeneity on $\mathrm{T} 1$ weighted images in MR, and rapidly increasing size. ${ }^{16,17}$

\section{Results}

\section{Patients}

Among 15 eligible patients, there were eight men and seven women (Table 1 ). The average age at diagnosis was 49 years (range: 18-83 years). Eleven patients had conventional MPNSTs, and 4 patients had MTT (27\%). The average age of the MPNST patients was 50 (range 18-83) while the average age of the MTT patients was 40 (range 27-67). Four patients (27\%) had NF1 (2 patients with MPNST, 2 patients with MTT).

Six tumors (40\%) were located in the mediastinum (two anterior, two middle and two in posterior mediastinum) (Figure 1), four $(27 \%)$ were in the chest wall (Figure 2), three (20\%) involved the lung, and two were paraspinal (13\%) (Figure 3).

Of the six mediastinal tumors, all were conventional MPNSTs and 2 were associated with NF1
(33\%). Of the four chest wall tumors, 2 were MTT, both of which were associated with NF1 (50\%), while the other two were conventional sporadic MNPST. Of the three lung tumors, 1 was a MTT (33\%); none of these patients had NF1. Of the two paraspinal tumors, 1 was a MTT (50\%) and none of the patients had NF1.

Nine patients presented with a chief complaint of pain, three with back pain, three with pleuritic chest pain, two with painful chest wall lumps, and one with right arm pain. Six patients presented with dyspnea or respiratory distress. One patient presented with right arm tingling/swelling. Table 2 summarizes the clinical presentation of the patients with intrathoracic MPNST. Only one patient was asymptomatic, with a large subxiphoid mass detected on surveillance imaging; the patient had received chemotherapy and radiation for Hodgkin's lymphoma 14 years prior to developing an MPNST. Clinical information regarding presentation was unavailable for one patient.

\section{Imaging features of primary tumors}

All patients had a single tumor representing MPNST. Average tumor size was $11 \mathrm{~cm}$ (range 3-32 $\mathrm{cm}$ ) (Table 3). Patients with MTT tended to have larger tumors at diagnosis (mean size $15 \mathrm{~cm}$ ) than those with conventional MPNSTs (mean size $9 \mathrm{~cm}$ ). However, this difference could not be statistically assessed due to a small sample size.

On CT, eight tumors (53\%) had lower attenuation than muscle and seven (47\%) were isoattenuating. On MRI, all five masses were isointense to muscles on T1-weighted images and hyperintense on T2-weighted images. Ten masses demonstrated mild enhancement, four had moderate enhancement, and one patient only had unenhanced CT. Eight (53\%) masses were heterogeneous and seven $(47 \%)$ were homogeneous.

Calcifications were seen in four lesions $(27 \%)$ and four $(27 \%)$ had features suggestive of necrosis. Three patients had punctate calcifications and one had chunky, nodular calcifications; calcifications were peripheral in two patients, central in one patient, and diffusely scattered in one. Of the four masses with appearance suggestive of necrosis on imaging, two had necrosis on pathology and one had cystic degeneration. No necrosis or cystic degeneration was noted on pathology in the fourth patient. Hemorrhage was seen in two masses $(13 \%)$.

The four masses that underwent PET/CT were intensely FDG_avid with mean $\mathrm{SUV}_{\max }$ of 10.5 
TABLE 1. Clinical features of patients with malignant peripheral nerve sheath tumor (MPNST) of chest

\begin{tabular}{|c|c|c|c|c|c|c|c|}
\hline $\begin{array}{l}\text { Sr } \\
\text { No }\end{array}$ & Age & Sex & NF1 & Location & Size $(\mathrm{cm})$ & Histology (MPNST/MTT) & Management \\
\hline 1 & 38 & $\mathrm{~F}$ & Yes & Middle mediastinum & 16 & MPNST & Chemoradiation \\
\hline 2 & 31 & M & No & Posterior mediastinum & 14 & MPNST & $\begin{array}{l}\text { Neoadjuvant chemoradiation, } \\
\text { followed by surgery }\end{array}$ \\
\hline 3 & 48 & M & No & Anterior mediastinum & 13.6 & MPNST & Chemoradiation \\
\hline 4 & 18 & $\mathrm{~F}$ & Yes & Middle mediastinum & 8 & MPNST & $\begin{array}{l}\text { Neoadjuvant chemoradiation, } \\
\text { followed by surgery }\end{array}$ \\
\hline 5 & 65 & M & No & Posterior mediastinum & 5.5 & MPNST & Neoadjuvant chemotherapy and surgery \\
\hline 6 & 33 & $\mathrm{~F}$ & No & $\begin{array}{l}\text { Anterior mediastinum- } \\
\text { subxiphoid }\end{array}$ & 4 & MPNST & Surgery \\
\hline 7 & 83 & $\mathrm{~F}$ & No & Chest wall & 17 & MPNST & Surgery, followed by chemoradiation \\
\hline 8 & 60 & M & No & Chest wall & 3 & MPNST & Surgery \\
\hline 9 & 27 & M & Yes & Chest wall & 4 & MTT & Surgery, followed by chemoradiation \\
\hline 10 & 44 & $\mathrm{~F}$ & Yes & Chest wall & 32 & MTT & None \\
\hline 11 & 51 & $\mathrm{~F}$ & No & Left lung & 4.5 & MPNST & Neoadjuvant chemotherapy and surgery \\
\hline 12 & 63 & M & No & Left lung & unknown & MPNST & Chemotherapy \\
\hline 13 & 67 & M & No & Left lung & 12.8 & MTT & Surgery \\
\hline 14 & 58 & M & No & Paraspinal & 2.5 & MPNST & Chemoradiation \\
\hline 15 & 61 & $\mathrm{~F}$ & No & Paraspinal & 10 & MTT & Chemotherapy \\
\hline
\end{tabular}

(range 4.4-23.6). One of these had MTT, and we noticed that this tumor had higher $\mathrm{SUV}_{\max }$ (23.6) than those without MTT (mean 6.1, range 4.4-8.9). Again, this difference could not be statistically analyzed given the small number of patients with this rare entity.

Neuroforaminal extension was seen in one posterior mediastinum patient and one paraspinal patient. Two patients had evidence of rib erosion on imaging, while two patients had evidence of rib displacement. Of the six mediastinal cases, four had invasion or encasement of adjacent structures on imaging such as trachea, mediastinal great vessels and esophagus, and one had intraspinal extension. Of the chest wall cases, one had invasion of adjacent tissues $(4 \mathrm{~cm})$ and another large mass had extensive invasion and displacement of multiple adjacent structures. All three of the lung cases had invasion of nearby structures including chest wall, diaphragm and mediastinum. Of the paraspinal cases, one patient presented with lung metastases, and the other patient had invasion into paraspinal musculature and extrapleural fat.

\section{Management}

In general, surgery was the treatment of choice whenever possible. Large masses which were initially deemed unresectable received neoadjuvant chemotherapy or chemoradiation, following which they were surgically resected if there was response and if they were considered resectable. Patients with nonradical resection received adjuvant chemotherapy.

Two posterior mediastinal masses and one middle mediastinal mass were treated with neoadjuvant chemoradiation or chemotherapy followed by surgery. The patient with the $14 \mathrm{~cm}$ posterior mediastinal mass received concurrent chemotherapy (etoposide/ifosfamide) and radiation followed by surgical resection, as the tumor was initially deemed unresectable. One patient with middle mediastinal mass and another with an anterior mediastinal mass underwent chemoradiation alone as they were deemed unresectable. The patient with the anterior mediastinal mass received chemotherapy (etoposide/cisplatin) followed by radiation, the patient with the middle mediastinal mass received concurrent chemotherapy (adriamycin/ ifosfamide) and radiation. Another patient with a $4 \mathrm{~cm}$ subxiphoid mass was resected without neoadjuvant therapy.

Of the four patients with chest wall masses, the $4 \mathrm{~cm}$ invasive mass and another large mass $(17 \mathrm{~cm})$ underwent surgery and chemoradiation, and one small mass $(3 \mathrm{~cm})$ was treated with surgical resection. The patient with the $4 \mathrm{~cm}$ invasive mass initially underwent non-radical resection, followed 
TABLE 2. Initial presentation of patients with malignant peripheral nerve sheath tumor (MPNST) of the chest

\begin{tabular}{|c|c|c|c|c|}
\hline $\begin{array}{l}\text { Sr } \\
\text { No }\end{array}$ & Location & Size $(\mathrm{cm})$ & Histology (MPNST/MTT) & Presentation \\
\hline 1 & Middle mediastinum & 16 & MPNST & Right arm pain with radiation to axilla \\
\hline 2 & Posterior mediastinum & 14 & MPNST & Back pain \\
\hline 3 & Anterior mediastinum & 13.6 & MPNST & $\begin{array}{c}\text { Dyspnea, found to have mass with partial obstruction } \\
\text { of right mainstem bronchus }\end{array}$ \\
\hline 4 & Middle mediastinum & 8 & MPNST & Dyspnea/pleuritic chest pain and pleural effusion \\
\hline 7 & Chest wall & 17 & MPNST & Right arm tingling/swelling \\
\hline 8 & Chest wall & 3 & MPNST & Painful chest wall lump \\
\hline 9 & Chest wall & 4 & MTT & Painful chest wall lump \\
\hline 10 & Chest wall & 32 & MTT & Dyspnea/pleuritic chest pain and pleural effusion \\
\hline 13 & Left lung & 12.8 & MTT & Unknown \\
\hline 14 & Paraspinal & 2.5 & MPNST & Back pain \\
\hline 15 & Paraspinal & 10 & MTT & Lung mets and dyspnea on exertion \\
\hline
\end{tabular}

MTT = malignant triton tumors; $\mathrm{Sr} \mathrm{No}=$ serial number

by radiation therapy, re-resection, and then chemotherapy with vincristine/actinomycin/cyclophosphamide/mesna; exact details of chemotherapy were not available for the $17 \mathrm{~cm}$ chest wall mass as patient received chemotherapy at an outside institution. The one patient with the $32 \mathrm{~cm}$ mass and extensive invasion of adjacent structures died prior to any treatment.

Of the three lung tumors, one case with mediastinal invasion was treated with neoadjuvant chemotherapy followed by surgery, one case with a chest wall and diaphragmatic invasion was treated with chemotherapy, and a hilar mass with invasion of the pulmonary artery was treated with total pneumonectomy.

Of the two paraspinal cases, one patient was treated with chemoradiation (further details not available), while the other was treated with chemotherapy alone, as the patient presented initially with widespread metastatic disease.

\section{Discussion}

Thoracic MPNSTs may involve mediastinum, lung or chest wall, with those in the mediastinum or chest wall manifesting as large elongated masses. They are often heterogeneous on CT and MRI, and intensely FDG-avid on PET. To our knowledge, this is the largest study of imaging findings of intrathoracic MPNST.

MPNSTs are rare, aggressive soft tissue sarcomas occurring anywhere in the body. They often occur in association with NF1, with an incidence ranging from $20-30 \% .1,3,5$ Four patients in our study had NF1 (27\%); this number is consistent with the reported incidence. MPNSTs most commonly occur in the extremities; intrathoracic MPNST are rarely reported, mostly in the form of isolated case reports. If MPNSTs arise in the nerves of the deep tissue, as occurs frequently with thoracic tumors, early diagnosis is clinically more difficult. ${ }^{4}$ Pain was the most common complaint and presenting symptom in others (7/15 patients). In a recent series of 175 patients with MPNSTs conducted at Mayo Clinic, the median tumor size was $6.0 \mathrm{~cm}^{1}$; our average tumor size was $11 \mathrm{~cm}$.

MTT, defined by the presence of rhabdomyoblasts in an MPNST on pathology, account for < $10 \%$ of MPNSTs. ${ }^{6}$ MTTs behave more aggressively than conventional MPNSTs. Out of the 15 patients in our study, 4 had the MTT subtype (27\%). These patients had a larger tumor size at diagnosis, measuring $15 \mathrm{~cm}$ compared to $9 \mathrm{~cm}$ for conventional MPNSTs.

MPNSTs typically are ovoid, occurring along a nerve $^{18}$, and presenting as a non-specific soft tissue mass. MPNSTs have low attenuation on CT. ${ }^{9}$ 

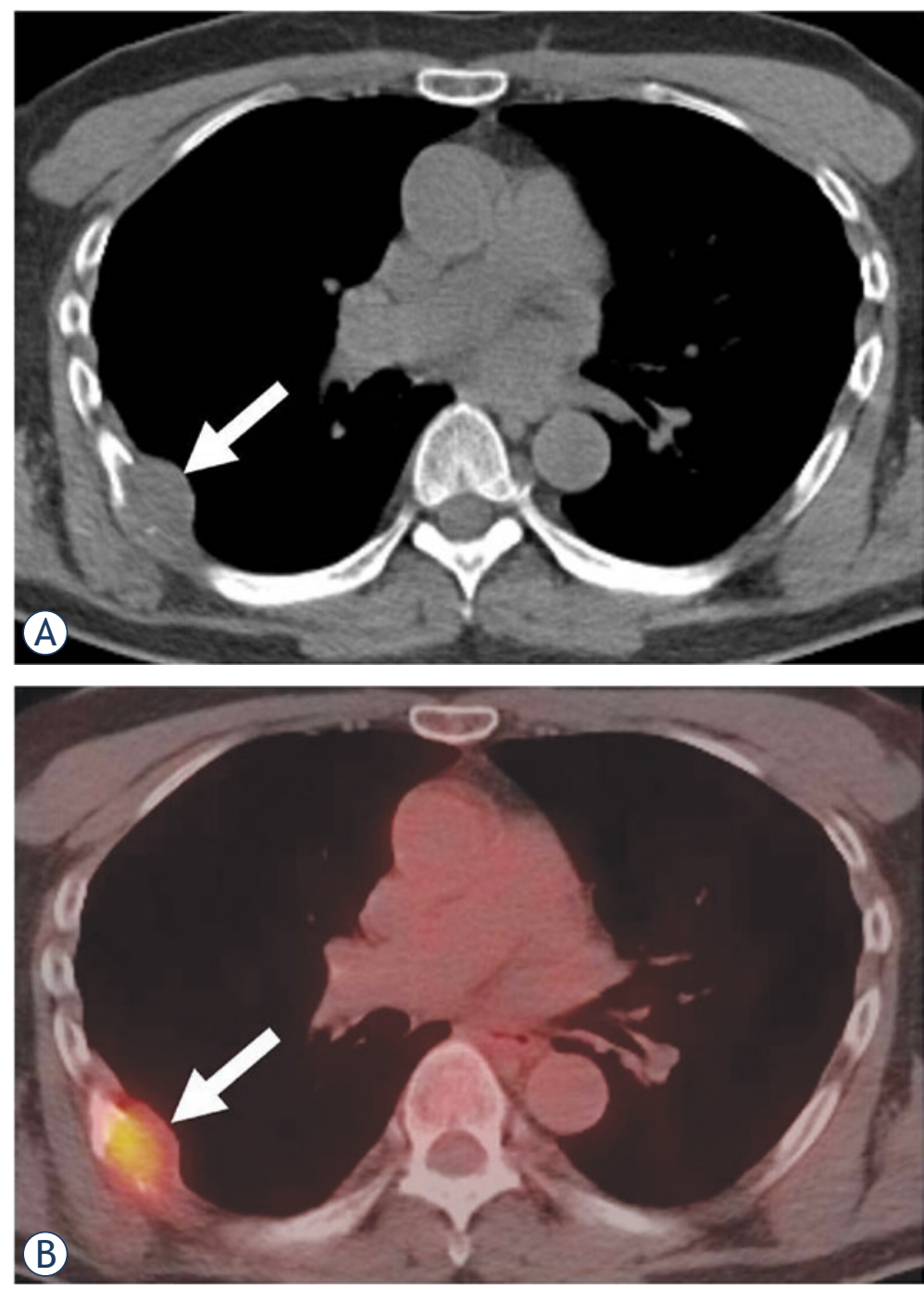

FIGURE 3. A 60-year-old man with left chest wall MPNST. A. Axial unenhanced CT image shows a low attenuation right chest wall mass abutting a rib (arrow) causing rib destruction and adjacent pleural thickening. B. Axial fused PET/CT image shows presence of moderate fluorodeoxyglucose (FDG) uptake (arrow, standardized uptake value [SUV] $\left.]_{\max } 4.4\right)$.

On MR, MPNSTs are isointense on T1 and hyperintense on T2. ${ }^{8}$ Our findings for intrathoracic MPNST are similar, as $8 / 15$ patients had hypoattenuating lesions on $\mathrm{CT}$, and of the five patients with $\mathrm{MR}$, all had isointense lesions on T1 and hyperintense lesions on T2. MPNSTs present as FDG-avid masses and can therefore be evaluated using PET/CT. ${ }^{19}$

Differentiation between malignant and benign nerve sheath tumors is challenging, especially in NF1 patients. Signs of MPNST on imaging include high attenuation and necrosis/hemorrhage on CT, heterogeneity on T1 weighted images in MR, and rapidly increasing size. ${ }^{16,17}$ A "target sign", or a central hypodense region on T2-weighted im- ages, was previously implicated as an indicator for MPNST, but this has not been corroborated by other groups.,20,21 Our data also did not confirm this finding, as a target sign was only seen in one patient. MPNST should be considered in the differential diagnosis for large thoracic masses found on imaging, especially if they are located along major nerves. MPNST should be especially high on the differential diagnosis if a thoracic mass is found in a patient with NF1.

Treatment of MPNST relies primarily on surgical resection. ${ }^{4}$ Neoadjuvant chemoradiation is often used for initially unresectable tumors with an intention to downstage the mass pre-resection. Radiation therapy can be given in addition to surgical resection for improved local control. ${ }^{5}$ Chemotherapy is typically reserved for aggressive cases, and also when there is tumor rupture, positive margins, or metastases, although improvement in survival has not been demonstrated. ${ }^{1}$ In an analysis of MPNST patients who received either surgery alone or multimodality treatment, the rate of recurrence was not significantly affected ${ }^{7}$, however, given the aggressive nature of MPNSTs, multimodality treatment is typically recommended. To the best of our knowledge, there are no studies comparing the efficacy of sequential versus concurrent chemoradiation in the treatment of MPNSTs.

Imaging assists with management of these patients, as tumors visualized to have invasion or compression of nearby structures often undergo additional therapy prior to surgical resection. PET/ CT may play an important role in MPNST management, as increased FDG uptake is associated with malignant transformation, and PET/CT has 72\% specificity for diagnosis of malignancy. ${ }^{19}$ In addition, whole-body MRI adequately assesses tumor burden in patients with NF1, and can allow visualization of tumors not observed on physical examination. This technique allows close surveillance of neurofibromas in NF1 patients and may allow for earlier detection of malignant transformation. ${ }^{21,23}$

An important limitation to this study was small sample size. However, intrathoracic MPNST are rare, and there are no prior reports on imaging features of series of these tumors. Due to the retrospective nature of the study with patients undergoing imaging evaluation as clinically indicated, we do not have MRI or PET/CTs for all 15 patients. As these are rare tumors, there is no fixed protocol for imaging evaluation of these masses.

In summary, intrathoracic MPNST may involve the mediastinum, lung and chest wall; mediastinal and chest wall tumors are usually large 
TABLE 3. Imaging features of patients with malignant peripheral nerve sheath tumor (MPNST) of the chest

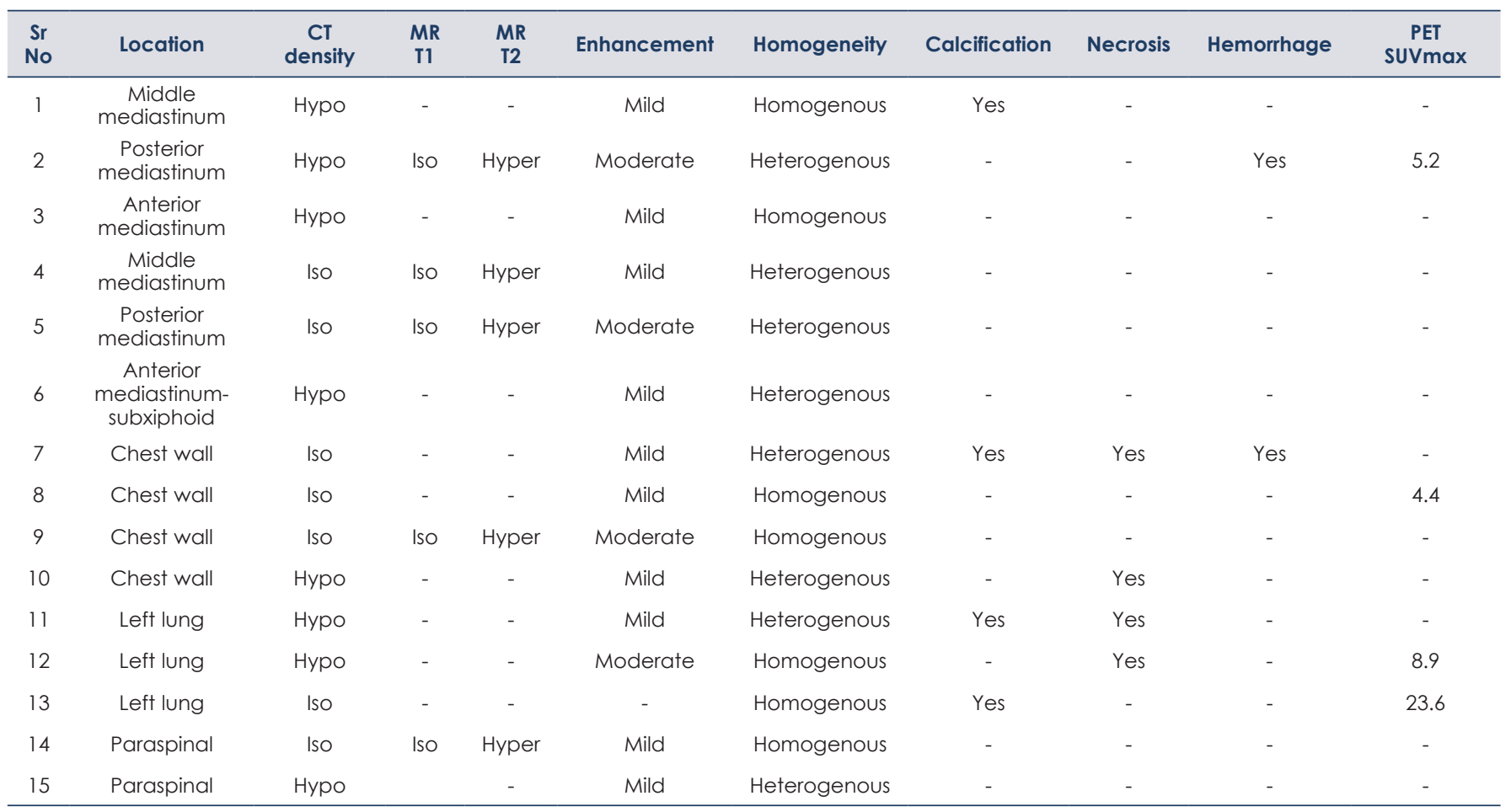

$\mathrm{Sr}$ No = serial number; $\mathrm{SUV}_{\max }=$ standardized uptake value

elongated masses along the course of nerves. They are usually hypoattenuating on $\mathrm{CT}$, hyperintense on T2-weighted images, are often heterogeneous, and are intensely FDG-avid masses. Comparison with previously published data on MPNSTs suggests that intrathoracic MPNST are similar in appearance to MPNSTs in other locations, but these tumors may present larger in size. For MPNSTs, imaging is often nonspecific, and although we as radiologists can raise the possibility, an accurate diagnosis always needs histologic confirmation. MPNST should be considered in the differential diagnosis when a large, elongated intrathoracic mass is found. The role of the radiologist lies in identification of invasion of adjacent structures as patients with invasion receive neoadjuvant or adjuvant chemoradiation.

\section{References}

1. Stucky CC, Johnson KN, Gray RJ, Stucky CC, Johnson KN, Gray RJ, et al. Malignant peripheral nerve sheath tumors (MPNST): the Mayo Clinic experience. Ann Surg Oncol 2012; 19: 878-85.

2. Van Herendael BH, Heyman SR, Vanhoenacker FM, De Temmerman G, Bloem JL, Parizel PM, et al. The value of magnetic resonance imaging in the differentiation between malignant peripheral nerve-sheath tumors and non-neurogenic malignant soft-tissue tumors. Skeletal Radiol 2006; 35: 745-53.
3. Ramanathan RC, Thomas JM. Malignant peripheral nerve sheath tumours associated with von Recklinghausen's neurofibromatosis. Eur J Surg Oncol 1999; 25: 190-3.

4. Shimizu J, Arano Y, Murata T, Ishikawa N, Yachi T, Nomura T, et al. A case of intrathoracic giant malignant peripheral nerve sheath tumor in neurofibromatosis type I (von Recklinghausen's disease). Ann Thorac Cardiovasc Surg 2008; 14: $42-7$.

5. Anghileri M, Miceli R, Fiore M, Mariani L, Ferrari A, Mussi C, et al. Malignant peripheral nerve sheath tumors: prognostic factors and survival in a series of patients treated at a single institution. Cancer 2006; 107: 1065-74.

6. McConnell YJ, Giacomantonio CA. Malignant triton tumors-complete surgical resection and adjuvant radiotherapy associated with improved survival. J Surg Oncol 2012; 106: 51-6.

7. Kamran SC, Howard SA, Shinagare AB, Krajewski KM, Jagannathan JP, Hornick JL, et al. Malignant peripheral nerve sheath tumors: Prognostic impact of rhabdomyoblastic differentiation (malignant triton tumors), neurofibromatosis 1 status and location. Eur J Surg Oncol 2013; 39: 46-52.

8. Kim JG, Sung WJ, Kim DH, Kim YH, Sohn SK, Lee KB. Malignant peripheral nerve sheath tumor in neurofibromatosis type I: unusual presentation of intraabdominal or intrathoracic mass. Korean J Intern Med 2005; 20: 100-4.

9. Chu YC, Yoon YH, Han HS, Han JY, Kim JM, Park IS. Malignant transformation of intrathoracic ancient neurilemmoma in a patient without von Recklinghausen's disease. J Korean Med Sci 2003; 18: 295-8.

10. McLaughlin EJ, Heuer GG, Whitmore RG, Birknes JK, Belasco J, Sterman $D$, et al. Treatment of a malignant peripheral nerve sheath tumor and its complications through a multidisciplinary approach. I Neurosurg Pediatr 2011; 7: 543-8.

11. Patel VS, St Louis JD, Oduntan O, Landolfo KP. Intrathoracic peripheral nerve sheath tumors in patients with neurofibromatosis type 1 (von Recklinghausen disease). J Thorac Cardiovasc Surg 2006; 131: 736-7.

12. Park JH, Choi KH, Lee HB, Rhee YK, Lee YC, Chung MJ. Intrathoracic malignant peripheral nerve sheath tumor in von Recklinghausen's disease. Korean J Intern Med 2001; 16: 201-4. 
13. Chao BH, Stogner-Underwood KA, Kiev J, Smith TJ. Intrathoracic malignant peripheral nerve sheath tumor in neurofibromatosis 1. J Clin Oncol 2008; 26: $2216-8$.

14. Yamaguchi M, Yoshino I, Fukuyama S, Osoegawa A, Kameyama T, Tagawa T, et al. Surgical treatment of neurogenic tumors of the chest. Ann Thorac Cardiovasc Surg 2004; 10: 148-51.

15. Podobnik J, Kocijancic I, Kovac V, Sersa I. 3T MRI in evaluation of asbestosrelated thoracic diseases-preliminary results. Radiol Oncol 2012; 44: 92-6.

16. Levy AD, Patel N, Dow N, Abbott RM, Miettinen M, Sobin LH. From the archives of the AFIP: abdominal neoplasms in patients with neurofibromatosis type 1: radiologic-pathologic correlation. Radiographics 2005; 25: 455-80.

17. Murphey MD, Smith WS, Smith SE, Kransdorf MJ, Temple HT. From the archives of the AFIP. Imaging of musculoskeletal neurogenic tumors: radiologic-pathologic correlation. Radiographics 1999; 19: 1253-80.

18. Li CS, Huang GS, Wu HD, Chen WT, Shih LS, Lii JM, et al. Differentiation of soft tissue benign and malignant peripheral nerve sheath tumors with magnetic resonance imaging. Clin Imaging 2008; 32: 121-7.

19. Bredella MA, Torriani M, Hornicek F, Ouellette HA, Plamer WE, Williams Z, et al. Value of PET in the assessment of patients with neurofibromatosis type 1. AJR Am J Roentgenol 2007; 189: 928-35.

20. Wasa J, Nishida $Y$, Tsukushi $S$, Shido $Y$, Sugiura H, Nakashima $H$, et al. MR features in the differentiation of malignant peripheral nerve sheath tumors and neurofibromas. AJR Am J Roentgenol 2010; 194: 1568-74.

21. Bhargava R, Parham DM, Lasater OE, Chari RS, Chen G, Fletcher BD. MR imaging differentiation of benign and malignant peripheral nerve sheath tumors: use of the target sign. Pediatr Radiol 1997; 27: 124-9.

22. Mautner VF, Asuagbor FA, Dombi E, Fünsterer C, Kluwe L, Wenzel R, et al. Assessment of benign tumor burden by whole-body MRI in patients with neurofibromatosis 1. Neuro Oncol 2008; 10: 593-8.

23. Van Meerbeeck SF, Verstraete KL, Janssens S, Mortier G. Whole body MR imaging in neurofibromatosis type 1. Eur J Radiol 2009; 69: 236-42. 